\title{
Detection of deletions in de novo "balanced" chromosome rearrangements: Further evidence for their role in phenotypic abnormalities
}

Caroline Astbury, PhD ${ }^{1}$, Laurie A. Christ, BS ${ }^{1}$, David J. Aughton, $M D^{2}$, Suzanne B. Cassidy, $M D^{3}$, Arun Kumar, PhD ${ }^{4}$, Evan E. Eichler, $P h D^{1}$, and Stuart Schwartz, $P h D^{1}$

\begin{abstract}
Purpose: The purpose of this study was to test the hypothesis that deletions of varying sizes in de novo apparently balanced chromosome rearrangements are a significant cause of phenotypic abnormalities. Methods: A total of fifteen patients, with seemingly balanced de novo rearrangements by routine cytogenetic analysis but with phenotypic anomalies, were systematically analyzed. We characterized the breakpoints in these fifteen cases (two of which were ascertained prenatally), using a combination of high-resolution GTG-banding, fluorescence in situ hybridization (FISH) with bacterial artificial chromosomes (BACs), and data from the Human Genome Project. Results: Molecular cytogenetic characterization of the 15 patients revealed nine with deletions, ranging in size from 0.8 to $15.3 \mathrm{Mb}$, with the number of genes lost ranging from 15 to 70 . In five of the other six cases, a known or putative gene(s) was potentially disrupted as a result of the chromosomal rearrangement. In the remaining case, no deletions were detected, and no known genes were apparently disrupted. Conclusions: Our study suggests that the use of molecular cytogenetic techniques is a highly effective way of systematically delineating chromosomal breakpoints, and that the presence of deletions of varying size is an important cause of phenotypic abnormalities in patients with "balanced" de novo rearrangements. Genet Med 2004:6(2):81-89.
\end{abstract}

Key Words: cryptic deletions, balanced chromosomal rearrangements, phenotypic abnormalities, BACs, FISH

Balanced chromosomal rearrangements, in which there is no cytogenetically detectable loss or gain of genetic material, may be either familial or de novo. The incidence of structural balanced rearrangements is thought to be 1 in 500 live births. ${ }^{1}$ Warburton ${ }^{2}$ predicted that the combined risk of associated phenotypic abnormalities for de novo reciprocal translocations and inversions was approximately $6.7 \%$. An increasing number of apparently "balanced" de novo chromosomal rearrangements have been associated with phenotypic abnormalities in recent years. ${ }^{3-5}$ A number of hypotheses have been postulated as to the cause of the phenotypic abnormalities. These include (1) a cryptic deletion undetected cytogenetically, causing the loss of a gene or genes, ${ }^{6,7}(2)$ a break in a gene at the translocation or inversion breakpoint, leading to loss of function, ${ }^{8-10}$ (3) position effects due to the new cytogenetic rearrangement, ${ }^{11,12}$ or (4) uniparental disomy, particularly in chromosomes known to be affected by imprinting. ${ }^{13,14}$ In addition, the balanced chromosome rear-

\footnotetext{
From the ${ }^{1}$ Center for Human Genetics and Department of Human Genetics, Case Western Reserve University School of Medicine and University Hospitals of Cleveland, Cleveland, Ohio; ${ }^{2}$ William Beaumont Hospital, Royal Oak, Michigan; ${ }^{3}$ University of California Irvine Medical Center, Orange, California; and ${ }^{4} D M R D G$, Indian Institute of Science, Bangalore, India.

Stuart Schwartz, PhD, Center for Human Genetics Laboratory, 10524 Euclid Ave, Sixth Floor, Cleveland, $\mathrm{OH} 44106-9959$

Received: October 1, 2003.

Accepted: December 18, 2003.
}

DOI: 10.1097/01.GIM.0000117850.04443.C9 rangement may be a serendipitous event unrelated to the phenotypic findings in the patient.

The use of molecular cytogenetic techniques, in association with high-resolution GTG-banding, has permitted further investigation of cases of seemingly balanced chromosomal rearrangements with associated phenotypic abnormalities. Delineated from the Human Genome Project, the utilization of bacterial artificial chromosomes (BACs) in conjunction with fluorescence in situ hybridization (FISH) has enabled more in-depth characterization of a number of interesting cases, potentially providing phenotype/genotype correlations. In addition, the use of BACs and FISH may aid prenatal diagnosis of balanced chromosomal rearrangements, although time constraints still remain an issue in these cases.

Previously, in our laboratory, we had shown that two out of three seemingly balanced chromosomal rearrangements harbored cryptic deletions, which might explain the phenotypic abnormalities seen in the patients. ${ }^{7}$ We have now studied a total of fifteen patients with de novo chromosomal rearrangements that include translocations, inversions, and insertions, and present these cases in this article.

\section{SUBJECTS AND METHODS}

\section{Clinical subjects}

Table 1 summarizes the clinical findings of the 15 patients analyzed and their karyotypes established at the time of diag- 
Table 1

Patient clinical information and initial karyotype

\begin{tabular}{|c|c|c|c|}
\hline Patient & Initial karyotype & Ascertainment & Clinical information \\
\hline AA & $46, \mathrm{XX}, \mathrm{t}(8 ; 10)(\mathrm{q} 11.23 ; \mathrm{p} 11.22)^{a, b}$ & Postnatal & Developmental delay; ventricular septal defect; aorta coarctation; minor anomalies \\
\hline JB & $46, \mathrm{XX}, \operatorname{inv}(2)(\mathrm{p} 21 \mathrm{q} 31)^{b}$ & Prenatal & Abnormal hand; right radius curved and short \\
\hline HB & $46, \mathrm{XX}$ or $46, \mathrm{XX}, \operatorname{del}(18)(? \mathrm{q} 12.2 \mathrm{q} 21.3)^{a, b}$ & Postnatal & Developmental delay; hypotonia; subtle dysmorphic features \\
\hline $\mathrm{LC}$ & $46, \mathrm{XY}$ or $46, \mathrm{XY}, ? \operatorname{del}(20)(\mathrm{q} 13.1 \mathrm{q} 13.3)^{a, b}$ & Postnatal & Bilateral clubbed feet; hypotonia; dysmorphic ears; gross motor delay \\
\hline GF & $46, \mathrm{XY}, \mathrm{t}(2 ; 12 ; 5)(\mathrm{p} 11.1 ; \mathrm{p} 11.2 ; \mathrm{p} 14)^{a}$ & Postnatal & Developmental delay; mild microcephaly; epicanthus tarsalis; tapering fingers \\
\hline GG & $46, \mathrm{XX}, \mathrm{t}(\mathrm{X} ; 16)(\mathrm{q} 11.2 \sim \mathrm{q} 13 ; \mathrm{p} 13.1 \sim \mathrm{p} 13.3)$ & Prenatal & Polyhydramnios; severe hypotonia; poor respiratory effect; mild syndactyly; clinodactyly \\
\hline $\mathrm{JM}$ & $46, \mathrm{XY}, \mathrm{t}(4 ; 16)(\mathrm{p} 16.1 ; \mathrm{p} 13.1)^{a, b}$ & Postnatal & Developmental delay; polycystic kidneys; ventricular septal defect; pulmonary stenosis \\
\hline $\mathrm{AM}$ & $45, \mathrm{XX}, \operatorname{dic}(10 ; 22)(\mathrm{q} 26.3 ; \mathrm{p} 13)$ & Postnatal & Developmental delay; negative for Fragile X \\
\hline $\mathrm{MN}^{c}$ & $46, \mathrm{XY}, \operatorname{inv}(7)(\mathrm{q} 22.1 \mathrm{q} 32.1)^{b}$ & Postnatal & Mental retardation; developmental delay; short stature; microcephaly; dysmorphic features \\
\hline BS & $46, \mathrm{XX}, \mathrm{t}(2 ; 8)(\mathrm{q} 21.1 \sim \mathrm{q} 21.3 ; \mathrm{q} 22.1 \sim \mathrm{q} 22.3)^{a}$ & Postnatal & Global developmental delay; leftward deviation of occiput and eyes; hypertonia; head lag \\
\hline $\mathrm{WS}^{c}$ & $46, \mathrm{XY}, \mathrm{t}(2 ; 6)(\mathrm{p} 22.2 ; \mathrm{q} 23.1)^{a, b}$ & Postnatal & Tetralogy of Fallot; coronal hypospadias; dysmorphic facies \\
\hline LS & $46, \mathrm{XY}, \mathrm{t}(6 ; 17)(\mathrm{q} 27 ; \mathrm{q} 24.3 \sim 25.1)^{a}$ & Postnatal & Congenital anomalies \\
\hline $\mathrm{CT}^{c}$ & $46, \mathrm{XX}, \mathrm{t}(2 ; 6)(\mathrm{q} 33.1 ; \mathrm{p} 12.2)$ & Postnatal & Mental and motor retardation; seizure disorder; bilateral aniridia; microcephaly \\
\hline LW & $46, \mathrm{XX}, \mathrm{t}(6 ; 10)(\mathrm{q} 21 ; \mathrm{q} 25.2)^{a}$ & Postnatal & Multiple anomalies; precocious puberty; mild to moderate mental retardation; exostoses \\
\hline $\mathrm{GM}^{d}$ & $\begin{array}{l}\text { 46,X,t }(\mathrm{X} ; 9)(\text { Xqter }>\text { Xp21.1 }:: 9 \mathrm{p} 22>9 \text { pter; } \\
\quad \text { 9qter }>9 \mathrm{p} 22:: \text { Xp21.2 }>\text { Xpter })\end{array}$ & Postnatal & Elevated CPK; EMG and muscle biopsy consistent with Duchenne muscular dystrophy \\
\hline
\end{tabular}

${ }^{a}$ Referral karyotype from another laboratory.

${ }^{b}$ Suspected deletion after high-resolution chromosome analysis in our laboratory that was later confirmed by FISH.

'These patients were originally described in Kumar. ${ }^{7}$

${ }^{d}$ Karyotype from Coriell Institute Cell Repository.

nosis. All the patients, except for patient GM, were either cases analyzed by our cytogenetic laboratory or referred to our laboratory for higher resolution chromosome analysis. Patient GM was analyzed, as only standard analysis had been done and molecular cytogenetics had not previously been used to characterize the breakpoints. The two prenatal cases (JB and GG) were ascertained due to abnormal ultrasounds. All of the postnatal patients were ascertained due to phenotypic abnormalities, which included developmental delay, multiple anomalies, and/or dysmorphic features. This group of patients, both preand postnatal, was chosen for analysis because they were believed to be more likely to have either a deletion or a potential gene disruption as the cause of their phenotypic abnormalities. All available patients in this group were analyzed.

\section{Cytogenetic analysis}

Metaphase chromosomes were prepared from peripheral blood lymphocytes, ${ }^{15}$ lymphoblasts, amniocytes, ${ }^{16}$ and/or fibroblast cultures, ${ }^{17}$ according to standard methods. Lymphocytes from peripheral blood samples were prepared in mitogen-stimulated (phytohemagglutinin and pokeweed) cultures to obtain high-resolution chromosomes. ${ }^{18}$ Chromosomes were GTG-banded, using standard methods, and at least 20 metaphase spreads were examined per patient. ${ }^{19}$ The resolution for the GTG-banded chromosomes was between 650 and 850 bands.

\section{Lymphoblast cell lines}

Lymphoblast cell lines were established according to standard methods, using Marmoset Epstein-Barr virus, phytohemagglutinin, and interleukin-2..$^{20}$ Due to the large number of FISH analyses on patient material, slides from these lymphoblast cell lines were used in the majority of cases to ensure enough material would be available.

\section{Replication analysis}

Replication analysis of the X chromosomes in patient GG was performed according to standard methods. ${ }^{21}$

\section{Molecular analysis}

All of the bacterial artificial chromosomes (BACs) used in these studies were obtained from a Human BAC filter library (RPC1-11) from the Roswell Park Cancer Institute (http:// genomics.roswellpark.org/human/overview.html). BACs were selected using the genome browser available from the University of California at Santa Cruz (http://genome.cse.ucsc.edu). During the course of this study, several different freezes were utilized, including data from October 2000 to April 2003.

BAC DNA was isolated using the Qiagen Plasmid Purification kit (Qiagen Inc., Valencia, CA). The yield of DNA was determined by UV spectrophotometry, and approximately 0.5 $\mu \mathrm{g}$ of BAC DNA was used for each FISH-labeling experiment. 


\section{Fluorescence in situ hybridization (FISH)}

BACs were labeled either indirectly (with digoxigenin, using the Bionick Labeling kit, Gibco-BRL, Gaithersburg, MD) or directly (with either Spectrum Orange or Spectrum Green, Nick Translation Labeling kit, Vysis Inc., Downers Grove, IL). FISH was performed according to the Oncor protocol (indirect labeling) or the Vysis protocol (direct labeling), both adapted from standard techniques. ${ }^{22}$ FISH images were captured on a Leica DMRB fluorescent microscope and analyzed with Applied Imaging software (Cytovision 2.7). For each FISH probe, at least five metaphase spreads were captured and analyzed.

\section{Human genome browser}

As stated above, BACs were selected using the UCSC genome browser. For all patients, the breakpoints determined by high-resolution chromosome analysis were used to select the cytogenetic bands of interest listed in the browser. Within each band, the selection of four to six BACs was decided, using the unique accession number assigned to each BAC under the full coverage option. Once these BACs had been tested, further BACs distal or proximal to the original selection were chosen. After a breakpoint and/or deletion had been delineated by means of the presence or absence of FISH signals or the split- ting of a FISH signal, then the number and type of genes within each breakpoint was determined by means of the data listed under "Genes and Gene Prediction Tracks."

\section{RESULTS}

\section{Patient AA}

This 4-year-old female patient was referred with the karyotype of 46,XX,t(8;10)(q11.23;p11.22)de novo. High-resolution chromosome analysis (Fig. 1A) in our laboratory confirmed that this patient had a rearrangement involving the long arm of chromosome 8 and the short arm of chromosome 10 (Fig. 1B). There was a suspected region of deletion in the short arm of chromosome 10. FISH analysis with BACs confirmed that there was a deletion in 10p11.23-10p12.1 of approximately $7 \mathrm{Mb}$ (Fig. 1C). A total of 26 genes were found within this deleted region. FISH analysis also revealed that the proximal breakpoint on chromosome 10 was in 10p12.1 between BAC RP11-371A19 (present on the normal chromosome 10 and on the der (8) chromosome), and the adjacent BAC 426A21 (present only on the normal chromosome 10). The distal breakpoint on chromosome 10 was in 10p11.23, between BAC RP11-14C22 (present on the der(10) chromosome and
A

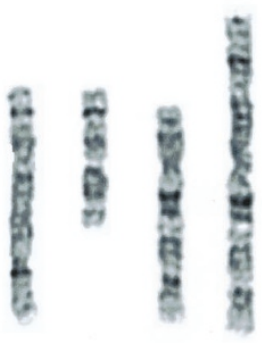

$8 \operatorname{der}(8) 10 \operatorname{der}(10)$

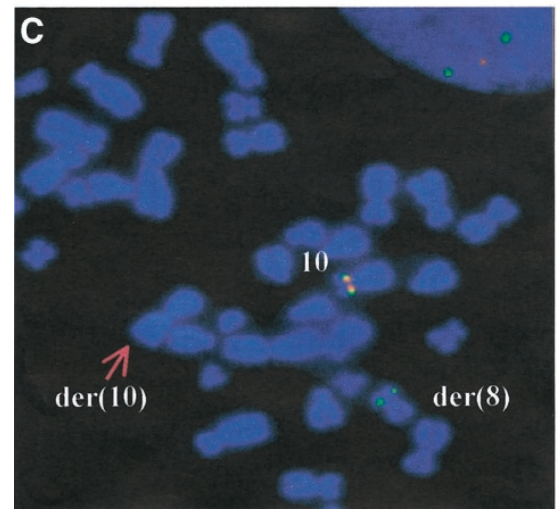

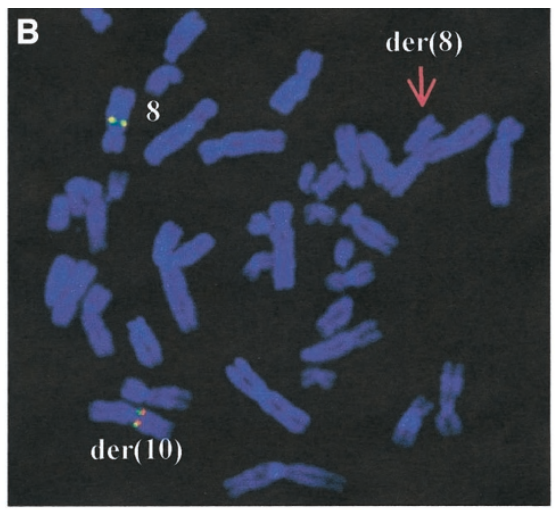
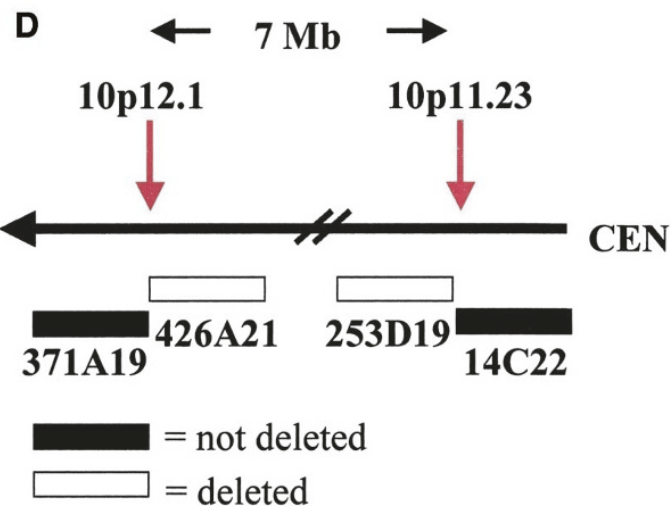

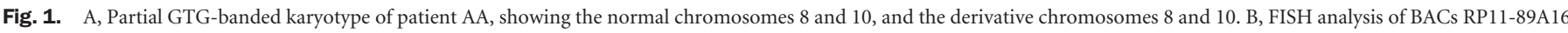

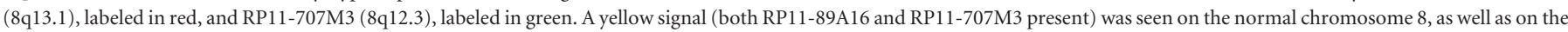

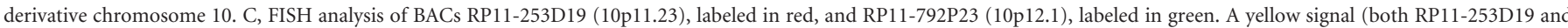

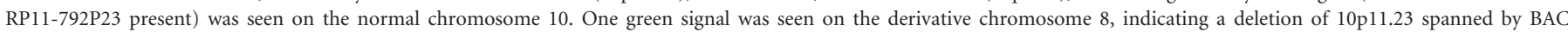

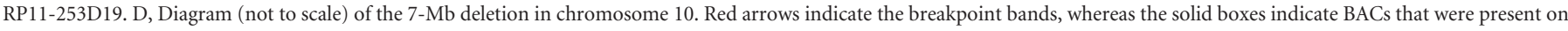
either the derivative chromosome 8 or the derivative chromosome 10. The clear boxes indicate BACs that were deleted on chromosome 10p. 
the normal chromosome 10), and the adjacent BAC RP11253D19, which was present only on the normal chromosome 10 (Fig. 1D). The breakpoint on chromosome 8 was in 8q12.3, between BAC RP11-16E8 (present on the normal chromosome 8 and the $\operatorname{der}(8)$ chromosome), and RP11-252M13 (present on the normal chromosome 8 and the der(10) chromosome). No genes were present in this region.

\section{Patient JB}

GTG-banded analysis of 20 metaphase cells from 17w3d amniocytes revealed, at low resolution, an apparently balanced pericentric inversion of chromosome 2 [46,XX,inv(2)(p21q31)]. Analysis of parental bloods determined this to be a de novo rearrangement. The GTG-banded results were confirmed by subsequent analysis of fibroblast tissue. However, at higher resolution, there was a suggestion of a deletion in $2 \mathrm{q} 31$. FISH analysis to define the breakpoints revealed a deletion from $2 \mathrm{q} 24.2$ to $2 \mathrm{q} 31.2$. The distal breakpoint was in 2q31.3 between BAC RP11-553I15 (present only on the normal chromosome 2), and the adjacent BACRP11-253H16 (present on both chromosomes 2). The proximal breakpoint was in 2q24.3 between BAC RP11-279B11 (one signal on the normal chromosome 2), and BAC RP11-646I19, present on both chromosomes 2 . The breakpoint delineation in this patient revealed a deletion of $15.3 \mathrm{Mb}$ of DNA. Within this deleted region, approximately 66 genes were located.

\section{Patient HB}

This 3-year-old female was referred with the karyotype of $46, \mathrm{XX}$ or $46, \mathrm{XX}, \operatorname{del}(18)(? \mathrm{q} 12.2 \mathrm{q} 21.3)$. High-resolution chromosome analysis in our laboratory revealed a more complex karyotype, involving an insertion of part of the long arm of chromosome 18 into the long arm of chromosome 11, and with a potential deletion in 18q12.2. The complexity and resolution of the abnormality in this patient are discussed in more detail in a separate article. ${ }^{23}$ Briefly, two nonconsecutive deletions in 18q were delineated (18q12.22-18q21.1 and 18q21.22$18 \mathrm{q} 22.2$ ), with the loss of $11 \mathrm{Mb}$ and $6.5 \mathrm{Mb}$ of DNA, respectively, as well as two nonconsecutive regions of $18 \mathrm{q}$ insertion into 11q.

\section{Patient LC}

This 6-month-old boy was referred with the karyotype of $46, \mathrm{XY}$ or $46, \mathrm{XY}$,?del(20)(q13.1q13.3). High-resolution chromosome analysis revealed a male karyotype with a pericentric inversion of chromosome 20, with the suggestion of a deletion in 20p12.3 [46,XY,der(20)inv(20)(p12.3q13.13)del(20)(p12.3p12.3)]. Analysis of parental bloods determined that the pericentric inversion of chromosome 20 was de novo. Subsequent BAC FISH analysis revealed a deletion of approximately $4.6 \mathrm{Mb}$ between bands 20p13 and 20p12.3, with the loss of about 20 genes. Two signals for BAC RP11-119B16 (20p13) were seen at the distal breakpoint, whereas only one signal for BAC RP11352D3 (20p13) was seen. BAC RP11-79E16 and RP11-430K20 were used to delineate the proximal breakpoint in 20p12.3, revealing only one signal for BAC RP11-79E16 (Fig. 2A) and
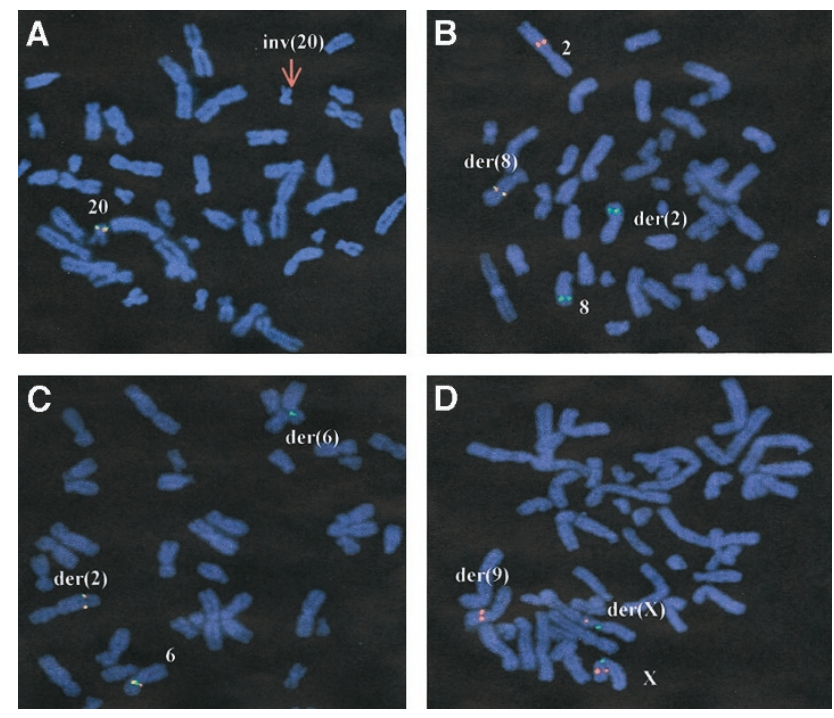

Fig. 2. A, Patient LC, FISH analysis of BACs RP11-79E16 (green) and RP11-19D2 (red), both in 20p12.3. Only one yellow signal was seen on the normal chromosome 20, indicating both BACs were deleted in the inverted chromosome 20. B, Patient BS, FISH analysis of BACs RP11-375H16 (2q23.2), in red, and RP11-795M12 (8q23.3), in green. A red signal (RP11-375H16) was present on the normal chromosome 2, whereas a green signal (RP11-795M12) was present on the normal chromosome 8. Yellow signals were seen on the derivative chromosomes 2 and 8 , indicating that these BACs spanned the translocation breakpoints in the derivative chromosomes. C, Patient CT, FISH analysis of BACs RP11-747O7 (6p12.3), in red, and RP11-529A12 (also in 6p12.3), in green. Both BACs were present on the normal chromosome 6 (yellow signal). RP11-747O7 was present on the derivative chromosome 2, and RP11-529A12 was present on both derivative chromosomes, indicating that this BAC spanned the translocation breakpoint. D, Patient GM, FISH analysis of BACs RP11-607K23 (Xp21.1), labeled in red, and RP11$461 \mathrm{~A} 12$ (Xq11), labeled in green. There was one red and one green signal on the normal $\mathrm{X}$ chromosome, whereas BAC RP11-607K23 (red) was split between the derivative X chromosome and the derivative chromosome 9.

two signals for BAC RP11-430K20, which was slightly centromeric to BAC RP11-79E16.

\section{Patient GF}

This 2-year-old boy was referred to our laboratory with the karyotype of $46, \mathrm{XY}, \mathrm{t}(2 ; 12 ; 5)(\mathrm{p} 11.1 ; \mathrm{p} 11.2 ; \mathrm{p} 14)$ de novo ish 22q11.2 (TUPLE1 $\times 2$ ). High-resolution chromosome analysis in our laboratory revealed a more complex karyotype, involving an insertion of part of the long arm of one chromosome 12 into the short arm of chromosome 5 , as well as a translocation between the same derivative chromosome 12 and one chromosome 2. Subsequent BAC FISH analysis confirmed the GTGbanded findings, as well as a cryptic deletion in the derivative chromosome 12 of approximately $3.6 \mathrm{Mb}$, beginning in 12 p 11.23 and extending to the centromere. However, the breakpoint delineation revealed an even greater complexity to the rearrangement, and this patient is discussed in more detail in a separate article. ${ }^{23}$

\section{Patient GG}

GTG-banded analysis of 20 metaphase cells from $17 \mathrm{w} 4 \mathrm{~d}$ amniocytes revealed an abnormal female karyotype (Fig. 3A), with an apparently balanced reciprocal translocation between the long arm of one chromosome $\mathrm{X}$ and the short arm of one 
A

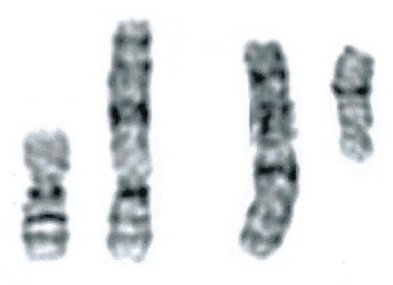

$16 \operatorname{der}(16) X \operatorname{der}(X)$

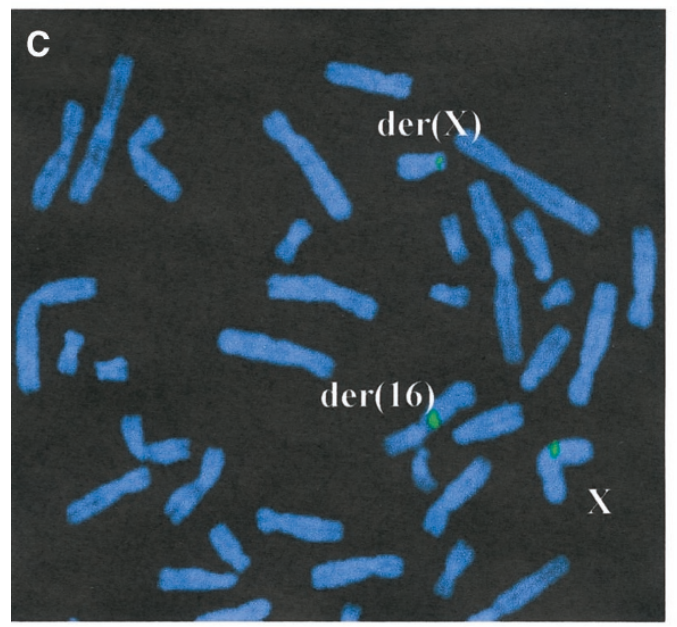

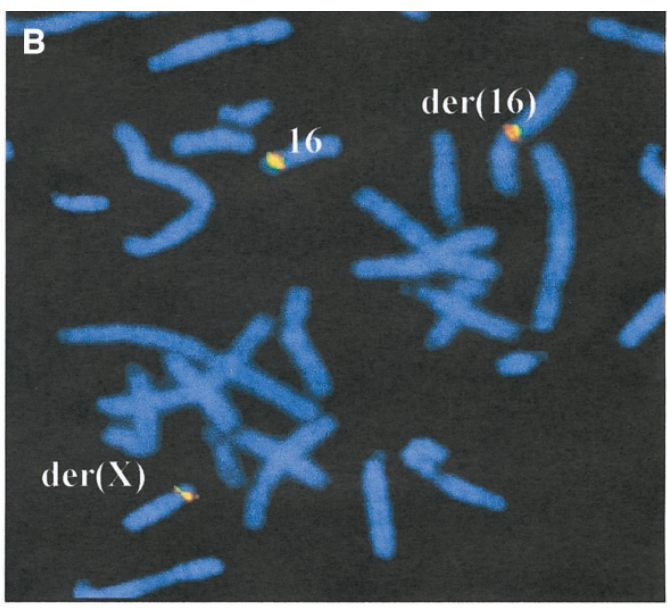

16p13.11

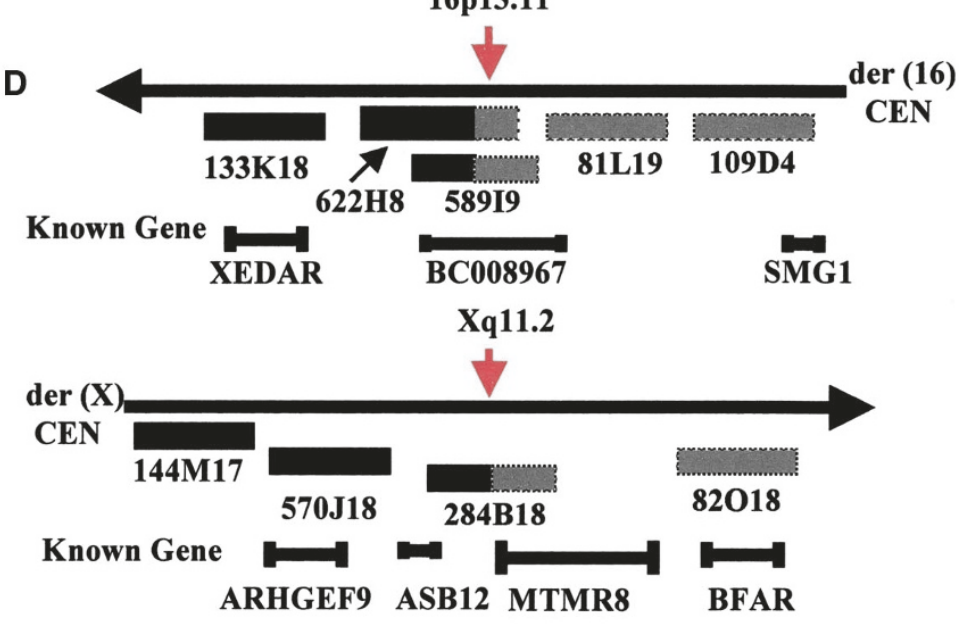

$=$ chromosome $16 \mathrm{BAC}$ present on $\operatorname{der}(16)$ or $\operatorname{der}(\mathrm{X})$ $=X$ chromosome BAC present on $\operatorname{der}(16)$ or $\operatorname{der}(\mathbf{X})$

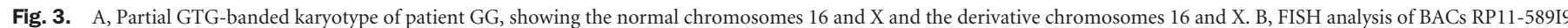

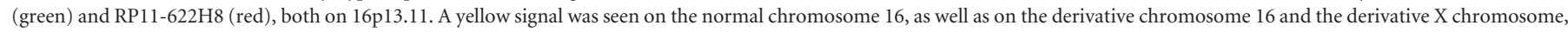

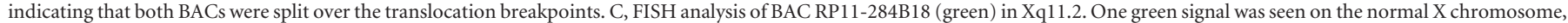

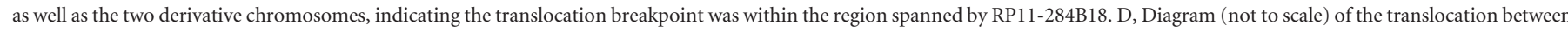

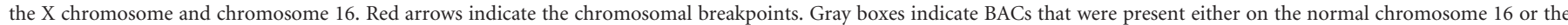

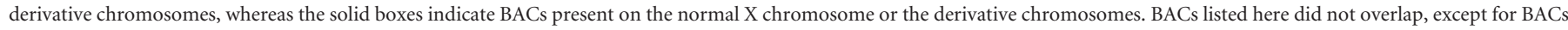
RP11-622H8 and RP11-589I9. Known genes (from the UCSC browser) are listed beneath the BACs.

chromosome $16 \quad[46, \mathrm{X}, \mathrm{t}(\mathrm{X} ; 16)(\mathrm{q} 11.2 \sim \mathrm{q} 13 ; \mathrm{p} 13.1 \sim \mathrm{p} 13.3)]$. Analysis of parental bloods revealed that the translocation was de novo. High-resolution chromosome analysis was also performed on a peripheral blood sample from this patient shortly after her birth, which confirmed the amniocyte findings. Replication analysis revealed that the normal $\mathrm{X}$ was late replicating in $100 \%(57 / 57)$ of cells analyzed, indicating completely skewed X inactivation. FISH studies with BAC RP11-589I9 and the adjacent BAC RP11-622H8, which both hybridized to 16p13.11, showed that these BACs were split between the derivative $\mathrm{X}$ chromosome and the derivative chromosome 16 (Fig. 3B). There was a hypothetical gene sequence, BC008967, present in this region of $16 \mathrm{p} 13.11$, the function of which might be disrupted. BAC RP11-284B18 at Xq11.2 was also split between the derivative chromosomes (Fig. 3C). BAC RP11-
284B18 spans part of the gene MTMR8 (myotubularin-related protein 8$)$; therefore, this gene might be disrupted in this patient (Fig. 3D).

\section{Patient JM}

This 2-year-old male was referred to our laboratory with the karyotype of 46,XY,t(4;16)(p16.1;p13.1). High-resolution chromosome analysis revealed that the karyotype was more complex, with a suspected translocation between chromosomes 4 and 17, and a potential deletion in the short arm of chromosome 16. Subsequent FISH analysis with subtelomere probes specific for $16 \mathrm{p}, 17 \mathrm{p}$, and $20 \mathrm{q}$ revealed that the subtelomeric region of chromosome $16 \mathrm{p}$ had translocated to $17 \mathrm{p}$, the subtelomeric region of $17 \mathrm{p}$ had translocated to $20 \mathrm{q}$, and the subtelomeric region of $20 \mathrm{q}$ had translocated to $16 \mathrm{p}$. In addi- 
tion, BAC FISH analysis revealed that a portion of $16 \mathrm{p}$ (16p13.12 to 16p12.2) had been inserted into 4p16.3. A cryptic deletion of $0.8 \mathrm{Mb}$ was found in 16p13.3, with the loss of approximately 50 genes. A more detailed analysis of this patient is presented in a separate article. ${ }^{23}$

\section{Patient AM}

High-resolution chromosome analysis of peripheral blood lymphocytes from this 5-year-old female revealed a karyotype of 45,XX,dic(10;22)(q26.3;p13)de novo. Even with GTGbanding at the 550 to 750 band level, no deletion could be conclusively identified. FISH analysis with a probe specific for the region above the $10 \mathrm{q}$ terminus (D10S2490) revealed that there had been a deletion of 10q26.3-10qter. According to the location of D10S2490 in the UCSC browser, this deletion was at least $4 \mathrm{Mb}$ in size, with the loss of approximately 8 genes. Unfortunately, lack of material precluded additional studies with BACs.

\section{Patient MN}

This patient was originally reported by our laboratory. ${ }^{7}$ Initial studies revealed that there was an apparently balanced, de novo paracentric inversion in the long arm of chromosome 7 $[46, \mathrm{XY}, \operatorname{inv}(7)(\mathrm{q} 22.1 \mathrm{q} 32.1)]$. FISH analysis of yeast artificial chromosomes (YACs) indicated the presence of a cryptic deletion near the proximal breakpoint of the inversion (7q22.1). The estimated size of the deletion was approximately $4 \mathrm{Mb}$. Subsequent BAC FISH analysis has shown that the deletion was about 3.1 Mb in size. Delineation of the proximal breakpoint in 7q22.1 was between BAC RP11-405I21 (not deleted) and BAC RP11-136B3 (deleted). The distal breakpoint was between BAC RP11-333G13 at 7q22.1 (only one signal was seen on the normal chromosome 7) and BAC RP11-163E9 at 7q22.1 (two signals seen). This cryptic deletion contained approximately 70 genes.

\section{Patient BS}

This 7-month-old female was referred with the karyotype of $46, \mathrm{XX}, \mathrm{t}(2 ; 8)$ (q21.1 q 21.3;q22.1 q22.3). High-resolution chromosome analysis in our laboratory confirmed this apparently balanced de novo rearrangement. Breakpoint delineation by FISH analysis of BACs was undertaken. The breakpoint in chromosome 2 was in 2q23.2, with BAC RP11-375H16 split between the derivative chromosome 2 and derivative chromosome 8. This BAC overlapped the gene MBD5 (methyl-CpG binding domain protein 5) which might, therefore, be disrupted. The breakpoint in chromosome 8 was in $8 \mathrm{q} 23.3$, with BAC RP11-795M12 split between the two derivative chromosomes (Fig. 2B). There were no known genes in this region, although there were two Genscan Gene Prediction sequences (NT_023811.50 and NT_023811.51).

\section{Patient WS}

This patient was originally reported by our laboratory, ${ }^{7}$ in which initial studies revealed a male karyotype with a de novo, apparently balanced rearrangement between the short arm of one chromosome 2 and the long arm of one chromosome 6 $[46, \mathrm{XY}, \mathrm{t}(2 ; 6)(\mathrm{p} 22.2 ; \mathrm{q} 23.1)]$. Microsatellite marker analysis was used to show that the patient was hemizygous for those markers mapping to 6q23.1. FISH analysis of YACs revealed that there was a deletion of approximately 4 to $6 \mathrm{Mb}$ between markers D6S412 and D6S1705. Subsequent BAC FISH analysis revealed that the deletion was larger than at first believed, at approximately $9 \mathrm{Mb}$, with the loss of about 17 genes, and the breakpoint was more proximal (in 6q22.31) than previously reported. Delineation of the proximal breakpoint in 6q22.31 was established using BAC RP11-428B11, for which only one signal was seen on the normal chromosome 6 , and STS marker D6S412 in 6q22.31 (one signal on the normal chromosome 6 and one on the derivative chromosome 6). Delineation of the distal breakpoint in 6q22.33 was established using BAC RP11$307 \mathrm{O} 14$ (deleted) and RP11-174C7 (not deleted). The breakpoint on chromosome 2 was not investigated, due to lack of material.

\section{Patient LS}

This 3-month-old boy was referred to our laboratory with the karyotype of 46,XY,t(6;17)(q27;q24.3 25.1) de novo. The karyotype was confirmed by high-resolution chromosome analysis. However, the GTG-banded analysis could not rule out a potential region of deletion in 17q24.3. BAC FISH analysis revealed that there was not a cryptic deletion at either of the two breakpoints. In addition, BAC RP11-270C4 in 6q27 was split between the der(6) and der(17) chromosomes. This BAC overlapped the gene SMOC2 (secreted modular calcium-binding protein 2). BAC RP11-203M16 in 17q24.3 was also split between the two derivative chromosomes. However, no genes were found in this region.

\section{Patient CT}

High-resolution chromosome analysis of this patient revealed the presence of a de novo, apparently balanced translocation between the long arm of chromosome 2 and the short arm of chromosome $6[46, \mathrm{XX}, \mathrm{t}(2 ; 6)(\mathrm{q} 33.1 ; \mathrm{p} 12.2)] .7$ Initial YAC studies suggested there was not a cryptic deletion in 2q33.1, as well as no evident deletion at the 6p12.2 breakpoint. Subsequent BAC FISH analysis of the $2 \mathrm{q} 33.1$ breakpoint revealed that BAC RP11-472L21 was present on both the normal chromosome 2 and on the der(2), whereas BAC RP11-505H14 (immediately adjacent to RP11-472L21) was found on the der(6), as well as the normal chromosome 2. There were no genes in this region. BAC RP11-529A21 in 6p12.3 was split between the derivative chromosome 6 and the derivative chromosome 2 (Fig. 2C). RP11-529A21 overlapped the potential gene sequence NT_007592.1262.

\section{Patient LW}

This 19-year-old female patient was referred with the karyotype of $46, \mathrm{XX}, \mathrm{t}(6 ; 10)(\mathrm{q} 21 ; \mathrm{q} 25.2)$. High-resolution chromosome analysis in our laboratory confirmed the translocation between one chromosome 6 and one chromosome 10. In addition, there was a pericentric inversion within the derivative 
chromosome 6. Due to the increased complexity of this de novo rearrangement, this patient will be discussed in more detail in a separate article. ${ }^{23}$ Briefly, although no deletions were found at any of the breakpoints, at least 14 genes spanned the breakpoint regions and, therefore, might have been disrupted.

\section{Patient GM}

This cell line (GM06007) was obtained from the Coriell Cell Repository at the Coriell Institute for Medical Research (http:// locus.umdnj.edu/nigms/nigms_cgi/display.cgi?GM06007).

The cell line was established from a 10-year-old female, who presented with Duchenne muscular dystrophy. ${ }^{24}$ Her karyotype was determined to be 46,X,t(X;9)(Xqter $>\mathrm{Xp} 21.1:: 9 \mathrm{p} 22>$ 9pter; 9qter $>$ 9p22::Xp21.2>Xpter). No GTG-banded studies were performed by our laboratory on this patient. FISH analysis of BACs from 9p22.2 revealed that BAC RP11-570H19 was present on the derivative chromosome $\mathrm{X}$ and the normal chromosome 9, whereas the adjacent BAC RP11-503K16 was present on both the normal chromosome 9 and the derivative chromosome 9. There were no genes present in this region. FISH analysis of BACs spanning the dystrophin gene at Xp21.1 revealed that BAC RP11-607K23 was split between the derivative $\mathrm{X}$ chromosome and the derivative chromosome 9 (Fig. $2 \mathrm{D})$; therefore, the dystrophin gene was disrupted in this patient.

\section{DISCUSSION}

The delineation and annotation of the Human Genome has resulted in the availability of bacterial artificial chromosomes (BACs), which have been instrumental in addressing the question of what may be occurring at the molecular level in apparently balanced chromosomal rearrangements in patients with phenotypic abnormalities. Previously, ${ }^{7}$ our laboratory had shown that two out of three phenotypically abnormal patients with seemingly balanced rearrangements possessed deletions that were not visible at the level of the microscope under routine analysis. In this study, we further characterize these three patients as well as 12 others believed to have balanced rearrangements (Table 2).

Nine of the fifteen patients we studied possessed deletions of varying size. As previously mentioned, two of these patients had been studied before, ${ }^{7}$ and the deletion breakpoints were further delineated in this study. The deletions in the remaining seven patients ranged from 0.8 to $15.3 \mathrm{Mb}$, with the loss of genes within these regions ranging from 15 to approximately 70 genes. High-resolution chromosome analysis of all the postnatal patients studied was used as a starting point, in the selection of BACs to delineate the breakpoints and to determine potential cryptic deletions. The normal limit of resolution for deletions at the microscope level is taken to be approximately 3 $\mathrm{Mb}$. In four of the nine patients, the deletions were $7 \mathrm{Mb}$ (patient $\mathrm{AA}$ ), $9 \mathrm{Mb}$ (patient WS), $11 \mathrm{Mb}$ (patient $\mathrm{HB}$ ), and 15.3 $\mathrm{Mb}$ (patient JB), all of which would have been supposed to have been visible microscopically. However, the difficulty in detecting the deletions in these rearrangements may be ac- counted for by the differences in DNA compaction, and by the altered banding patterns that result after a translocation, insertion, or inversion, as well as the level of resolution used to visualize the chromosomes. In patient WS, in whom a deletion of approximately $9 \mathrm{Mb}$ was seen in $6 \mathrm{q} 22.31$ to $6 \mathrm{q} 22.33$, the translocation was between the short arm of chromosome 2 and the long arm of chromosome 6 . These two chromosomes, at high resolution, are defined by a large number of bands and sub-bands; it may be that the very nature of the chromosomes involved in this translocation led to the difficulty of definitively determining that there had been a deletion using G-banding alone. This might also be the case for patient AA, in whom a deletion of $7 \mathrm{Mb}$ was seen, after a translocation between chromosomes 8 and 10. For patient HB, a deletion in one chromosome 18 was visible using G-banding; however, a second, cryptic deletion was only detected when the breakpoints in the rearrangement were delineated.

We found that a gene or potential gene(s) in five out of our fifteen patients may have been disrupted as a result of the patient's chromosomal rearrangement. Only one of these disruptions (of the dystrophin gene in patient GM) can be directly assigned to the observed phenotype in the patient (Duchenne muscular dystrophy). Initially, it was reported that no dystrophin gene deletion or duplication was detected by PCR analysis in this patient. ${ }^{24}$ Our analysis revealed that the gene had been split, as delineated by BAC RP11-607K23 in Xp21.1, signals for which were seen on the derivative $\mathrm{X}$ chromosome and the derivative chromosome 9. However, for the remaining four patients in whom a potential gene disruption was suggested, there is a need for further gene function tests, to assess the causality or coincidence of the potential gene disruption.

Currently, time constraints in the use of FISH with BAC probes are a problem in delineating prenatal cases. It may take up to three months to determine the breakpoints in a rearrangement, and longer with more complex rearrangements. More BACs, and consequently more time, are required to delineate an inversion or insertion versus a simple translocation, where only the absence or presence of a FISH signal is expected. For insertions or inversions, the relative position of FISH signals to each other must be assessed, which may be difficult with shorter, lymphoblast chromosomes. As more and more BAC probes become available, time and the number of BACs that need to be analyzed should become less of an issue. Our study emphasizes the use of BAC probes to analyze systematically prenatal, and postnatal, de novo rearrangements in order to present the parents with as much information as possible regarding phenotype/genotype correlations. However, although BAC FISH analysis may provide information regarding breakpoint delineation and/or the presence of deletions, or potential gene disruptions, there is a limit to the clinical application of the analysis, in that it cannot answer questions of the effects of chimeric genes or haploinsufficiency of genes.

There is sparse information in the literature about the frequency of deletions (or duplications) versus those with gene disruption in patients with seemingly balanced chromosome rearrangements and phenotypic abnormalities. From our data, 
Table 2

Summary of patient cytogenetic breakpoint information and UCSC coordinates

\begin{tabular}{|c|c|c|c|}
\hline Patient & $\begin{array}{l}\text { Cytogenetic } \\
\text { breakpoint }\end{array}$ & Deletion or potential gene disruption ${ }^{b}$ & UCSC coordinates $^{a}$ \\
\hline \multirow[t]{2}{*}{$\mathrm{AA}$} & $8 \mathrm{q} 12.3$ & Neither & $63,896,341$ \\
\hline & $10 \mathrm{p} 12.1-10 \mathrm{p} 11.23$ & Deletion (7 Mb) & $24,054,879-31,037,984$ \\
\hline JB & $2 \mathrm{q} 24.3-2 \mathrm{q} 31.3$ & Deletion (15.3 Mb) & $167,154,692-182,495,730$ \\
\hline \multirow[t]{2}{*}{$\mathrm{HB}$} & $18 \mathrm{q} 12.22-18 \mathrm{q} 21.1$ & Deletion (11 Mb) & $33,629,537-44,920,266$ \\
\hline & $18 \mathrm{q} 21.22-18 \mathrm{q} 22.2$ & Deletion $(6.5 \mathrm{Mb})$ & $61,085,105-67,558,355$ \\
\hline LC & 20p13-20p12.3 & Deletion (4.6 Mb) & $3,816,827-9,108,649$ \\
\hline GF & $12 \mathrm{p} 11.23$ & Deletion (3.6 Mb) & $29,911,562-33,543,861$ \\
\hline \multirow[t]{2}{*}{ GG } & $16 \mathrm{p} 13.11$ & Potential gene disruption (BC008967) & $15,466,460$ \\
\hline & $\mathrm{Xq} 11.2$ & Potential gene disruption (MTRM8) & $61,667,590$ \\
\hline JM & $16 \mathrm{p} 13.3$ & Deletion $(0.8 \mathrm{Mb})$ & $1,301,299-2,067,578$ \\
\hline $\mathrm{AM}$ & $10 \mathrm{q} 26.3$ & Deletion $(\approx 4 \mathrm{Mb})$ & No BACs analyzed \\
\hline MN & $7 \mathrm{q} 22.1$ & Deletion (3.1 Mb) & $98,426,024-101,573,162$ \\
\hline \multirow[t]{2}{*}{ BS } & $2 \mathrm{q} 23.2$ & Potential gene disruption (MBD5) & $147,670,251$ \\
\hline & $8 \mathrm{q} 23.3$ & Neither & $117,203,265$ \\
\hline WS & $6 \mathrm{q} 22.31-6 \mathrm{q} 22.33$ & Deletion $(9 \mathrm{Mb})$ & $120,553,426-129,928,010$ \\
\hline \multirow[t]{2}{*}{ LS } & $6 \mathrm{q} 27$ & Potential gene disruption (SMOC2) & $168,749,320$ \\
\hline & $17 \mathrm{q} 24.3$ & Neither & $70,045,173$ \\
\hline \multirow[t]{2}{*}{ CT } & $2 \mathrm{q} 33.1$ & Neither & $199,882,049$ \\
\hline & $6 \mathrm{p} 12.3$ & Potential gene disruption (NT_007592.1262) & $51,302,164$ \\
\hline \multirow[t]{3}{*}{ LW } & $6 \mathrm{p} 24.2$ & Potential gene disruption (GCNT2) & $10,639,333$ \\
\hline & $6 \mathrm{q} 21$ & Potential gene disruption (HDAC2) & $110,835,182$ \\
\hline & $10 \mathrm{q} 26.13$ & Potential gene disruption (GPR26) & $125,396,469$ \\
\hline \multirow[t]{2}{*}{ GM } & $9 \mathrm{p} 22.2$ & Neither & $18,646,167$ \\
\hline & $\mathrm{Xp} 21.1$ & Gene disruption (DMD) & $31,112,989$ \\
\hline
\end{tabular}

${ }^{a}$ Coordinates taken from April 2003 UCSC browser.

${ }^{b}$ Known gene in parenthesis (from UCSC browser).

it would appear that deletions occur more frequently than gene disruptions, in cases with a seemingly balanced chromosome rearrangement. However, there may be a bias of ascertainment in the selection of patients used in our study. We were either referred or restudied patients that were believed by clinicians to have a constellation of phenotypic findings consistent with a chromosomal abnormality. In contrast to these, patients with suspected gene disruptions are more likely to have a more syndromic phenotype. Therefore, by our referral patterns the overall frequency of deletions may be elevated.

One limitation of this study may be the inability to distinguish duplications of adjacent BAC signals, as opposed to a BAC signal split between two derivative chromosomes. In all of the patients we studied in which we detected a split FISH signal, the signal was approximately the same size on each of the derivative chromosomes. In addition, we generally analyzed BACs that were proximal or distal to the BAC that was split, and did not see a split signal in those BACs, suggesting that the BAC was split and not duplicated.
In summary, we have shown that 9 out of 15 patients with apparently balanced rearrangements and phenotypic abnormalities possessed deletions, some cryptic, whereas five patients had a potential gene disruption. This study emphasizes that it is essential to perform high-resolution chromosome analysis on all patients with de novo balanced rearrangements, as well as demonstrating the systematic utility of BAC probes by FISH. This study also highlights the symbiosis of traditional cytogenetic techniques with more modern molecular cytogenetic techniques.

\section{ACKNOWLEDGMENTS}

We gratefully acknowledge Michele Eichenmiller and Cassy Gulden, for their help with the BAC preparations, Bobbi Sundman for the lymphoblast cell cultures, Renee Slaw and the amnio group for the amniotic cell cultures, Bruce Tyler for the fibroblast cultures and harvest, and Steve Nagy for his slide preparation. 


\section{Electronic database information}

URLs for data presented are as follows:

UCSC Genome Bioinformatics: http://genome.cse.ucsc.edu Roswell Park Cancer Institute: http://genomics.roswellpark.org/human/overview.html Coriell Institute for Medical Research: http://locus.umdnj.edu/ nigms/nigms_cgi/display.cgi?GM06007

\section{References}

1. Hsu LYF. Prenatal diagnosis of chromosomal abnormalities through amniocentesis. In: Milunsky A, editor. Genetic disorders and the fetus: diagnosis, prevention and treatment. Baltimore: Johns Hopkins University Press, 1998:179-248.

2. Warburton D. De novo balanced chromosomal rearrangements and extra marker chromosomes identified at prenatal diagnosis: clinical significance and distribution of breakpoints. Am J Hum Genet 1991;49:995-1013.

3. Freeman SB, Muralidharan K, Pettay D, Blackston RD, May KM. Asplenia syndrome in a child with a balanced reciprocal translocation of chromosomes 11 and 20 [46,XX, t(11;20)(q13.1;q13.13)]. Am J Med Genet 1996;61:340-344.

4. Tar A, Ion A, Solyom J, Gyorvari B, Stephenson C, Barbaux S et al. Hypertelorism and hypospadias associated with a de novo apparently balanced translocation between 8q22.3-23 and 20p13. Am J Med Genet 1997;68:231-235.

5. Borg I, Squire M, Menzel C, Stout K, Morgan D, Willatt L et al. A cryptic deletion of 2q35 including part of the PAX3 gene detected by breakpoint mapping in a child with autism and de novo 2;8 translocation. J Med Genet 2002;39:391-399.

6. Puissant H, Azoulay M, Serre JL, Piet LL, Junien C. Molecular analysis of a reciprocal translocation t(5;11)(q11;p13) in a WAGR patient. Hum Genet 1988;79:280-282.

7. Kumar A, Becker LA, Depinet TW, Haren JM, Kurtz CL, Robin NH et al. Molecular characterization and delineation of subtle deletions in de novo 'balanced' chromosomal rearrangements. Hum Genet 1998;103:173-178.

8. Bugge M, Bruun-Petersen G, Brondum-Nielsen K, Friedrich U, Hansen J, Jensen G et al. Disease associated balanced chromosomal rearrangements: a resource for large scale genotype-phenotype delineation in man. J Med Genet 2000;37:858-865.

9. Duba HC, Doll A, Neyer M, Erdel M, Mann C, Hammerer I et al. The elastin gene is disrupted in a family with a balanced translocation $\mathrm{t}(7 ; 16)(\mathrm{q} 11.23 ; \mathrm{q} 13)$ associated with a variable expression of the Williams-Beuren syndrome. Eur J Hum Genet 2002;10:352-361.
10. Vervoort VS, Viljoen D, Smart R, Suthers G, DuPont BR, Abbott A et al. Sorting nexin 3 (SNX3) is disrupted in a patient with a translocation $\mathrm{t}(6 ; 13)(\mathrm{q} 21 ; \mathrm{q} 12)$ and microcephaly, microphthalmia, ectrodactyly, prognathism (MMEP) phenotype. J Med Genet 2002;39:893-899.

11. Fantes J, Redeker B, Breen M, Boyle S, Brown J, Fletcher J et al. Aniridia-associated cytogenetic rearrangements suggest that a position effect may cause the mutant phenotype. Hum Mol Genet 1995;4:415-422.

12. Sutherland HF, Wadey R, McKie JM, Taylor C, Atif U, Johnstone KA et al. Identification of a novel transcript disrupted by a balanced translocation associated with DiGeorge syndrome. Am J Hum Genet 1996;59:23-31.

13. James RS, Temple IK, Patch C, Thompson EM, Hassold T, Jacobs P. A systematic search for uniparental disomy in carriers of chromosomal translocations. Eur J Hum Genet 1994;2:83-95.

14. Robinson WP, Bernasconi F, Basaran S, Yukel-Apak M, Neri G, Serville F et al. A somatic origin of homologous Robertsonian translocations and isochromosomes. Am J Hum Genet 1994;54:290-302.

15. Moorhead PS, Nowell PC, Mellman WJ, Battips DM, Hungerford DA. Chromosome preparations of leukocytes cultured from human peripheral blood. Exp Cell Res 1960;20:613-616.

16. Hoehn H. Fluid cell culture. In: Milunsky A, editor. Genetic disorders and the fetus: Diagnosis, prevention and treatment. Baltimore: Johns Hopkins University Press, 1998:128-149.

17. Barch MJ, Knutsen T, Spurbeck, JL, editors. The ACT cytogenetics laboratory manual, 3rd ed. New York: Raven Press, 1997.

18. Yunis JJ. High resolution of human chromosomes. Science 1976;191:1268-1270.

19. Seabright M. A rapid banding technique for human chromosomes. Lancet 1971;2: 971-972.

20. Neitzel H. A routine method for the establishment of permanent growing lymphoblastoid cell lines. Hum Genet 1986;73:320-326.

21. Latt SA, Willard HF, Gerald PS. BrdU-33258 Hoechst analysis of DNA replication in human lymphocytes with supernumerary or structurally abnormal X chromosomes. Chromosoma 1976;57:135.

22. Pinkel D, Straume T, Gray JW. Cytogenetic analysis using quantitative, high-sensitivity, fluorescence hybridization. Proc Natl Acad Sci U S A 1986;83:2934-2938.

23. Astbury C, Christ LA, Aughton DJ, Cassidy SB, Fujimoto A, Pletcher BA et al. Delineation of complex chromosomal rearrangements: evidence for increased complexity. Hum Genet. In press.

24. Emanuel BS. Further evidence for Xp21 location of Duchenne muscular dystrophy (DMD) locus: X/9 translocation in a female with DMD. Am J Hum Genet 1981;33: 103A. 